\title{
Evidence of local moment formation in Co-based Heusler alloys
}

\author{
N. D. Telling, ${ }^{1,2}$ P. S. Keatley, ${ }^{3}$ G. van der Laan,,${ }^{2,4}$ R. J. Hicken, ${ }^{3}$ E. Arenholz, ${ }^{5}$ Y. Sakuraba, ${ }^{6}$ M. Oogane, ${ }^{7}$ Y. Ando, ${ }^{7}$ \\ K. Takanashi, ${ }^{6}$ A. Sakuma, ${ }^{7}$ and T. Miyazaki ${ }^{7}$ \\ ${ }^{1}$ School of Earth, Atmospheric and Environmental Science, University of Manchester, Oxford Road, Manchester M13 9P, \\ United Kingdom \\ ${ }^{2}$ Magnetic Spectroscopy Group, STFC Daresbury Laboratory, Warrington WA4 4AD, United Kingdom \\ ${ }^{3}$ School of Physics, University of Exeter, Stocker Road, Exeter EX4 4QL, United Kingdom \\ ${ }^{4}$ Diamond Light Source, Chilton, Didcot OX11 ODE, United Kingdom \\ ${ }^{5}$ Advanced Light Source, Lawrence Berkeley National Laboratory, Berkeley, California 94720, USA \\ ${ }^{6}$ Institute for Materials Research, Tohoku University, Katahira 2-1-1, Sendai 980-8577, Japan \\ ${ }^{7}$ Department of Applied Physics, Graduate School of Engineering, Tohoku University, Aoba-yama 6-6-05, Sendai 980-8579, Japan
}

(Received 18 August 2008; revised manuscript received 15 October 2008; published 25 November 2008)

\begin{abstract}
We examine the formation of local moments in Heusler alloys of the composition $\mathrm{Co}_{2} \mathrm{MnZ}$ (where $Z=\mathrm{Si}$ or $\mathrm{Al}$ ) using the combined techniques of $\mathrm{x}$-ray magnetic circular and linear dichroism. The existence of local moments in half-metallic Heusler alloys is reliant upon the band gap in the minority-spin states. By utilizing the element-specific nature of x-ray absorption techniques we are able to explore the degree of localization of moments on $\mathrm{Co}$ and $\mathrm{Mn}$ atoms. We observe a crucial difference in the localization of the Co moment when comparing $\mathrm{Co}_{2} \mathrm{MnSi}(\mathrm{CMS})$ and $\mathrm{Co}_{2} \mathrm{MnAl}$ films that is consistent with the predicted larger minority-spin gap in the Co partial density of states for CMS. These results provide important evidence for the dominant role of the Co minority-spin states in realizing half-metallic ferromagnetism in this system.
\end{abstract}

DOI: 10.1103/PhysRevB.78.184438

PACS number(s): 75.70.-i, 78.70.Dm, 72.25.-b, 75.47.-m

\section{INTRODUCTION}

Half-metallic ferromagnetism (HMF) is observed in materials where the majority-spin states have metallic character while the minority-spin band contains an energy gap at the Fermi level. ${ }^{1}$ This unique property leads in principle to $100 \%$ spin-polarization making these materials extremely attractive contenders for spintronic applications. A great deal of theoretical effort has been applied to understand the origin of band gaps in half-metallic ferromagnets such as the full Heusler alloys $\mathrm{Co}_{2} \mathrm{MnZ}$ (where $Z=\mathrm{Si}, \mathrm{Al}, \mathrm{Ge}, \mathrm{Sn}$, or $\mathrm{Ga}$ ). The band gap in the minority-spin states is thought to arise from the hybridization of Co and Mn $d$ orbitals. $^{2-4}$ The overall gap is determined by the Co-Co interaction while the "effective" gap in the Mn partial density of states (PDOS) is much larger as a result of the Co-Co-Mn hybridization. Typically, experimental results on these materials provide indirect evidence for half-metallic ferromagnetism (HMF) through the observation of large tunneling magnetoresistance (TMR). ${ }^{5-7}$ Thus a systematic study of the band gap in the PDOS for the different elements in these alloys is asked for. Although surface science techniques, such as spin-resolved photoemission, can directly probe the spin polarization at a half-metal surface, they cannot be used to access the crucial interface region of half-metal and barrier layer (typically alumina or $\mathrm{MgO}$ ) which is responsible for the TMR.

Kübler $e t \mathrm{al}^{8}{ }^{8}$ pointed out that a local exclusion of minority spin around $\mathrm{Mn}$ atoms in Heusler alloys leads to the formation of so-called "ideal" local moments. The existence of these local moments is reliant upon the band gap in the minority-spin states, i.e., the half-metallic character of the system. X-ray magnetic dichroism techniques could be a powerful tool for studying such systems due to their elementspecific sensitivity to the localization of magnetic moments.
Previously it has been shown that the existence of local moments gives rise to a very pronounced multiplet structure visible in the $\mathrm{x}$-ray magnetic circular dichroism (XMCD) spectra. ${ }^{9,10}$ In addition, the $\mathrm{x}$-ray magnetic linear dichroism (XMLD) effect is found to be several times larger in a local moment system than in an itinerant system. ${ }^{11,12}$

In this work we performed both XMCD and XMLD measurements on Heusler alloys of the form $\mathrm{Co}_{2} \mathrm{MnSi}$ (CMS) and $\mathrm{Co}_{2} \mathrm{MnAl}$ (CMA). By utilizing the element-specific nature of the techniques we were able to separately explore the degree of localization of moments on Co and $\mathrm{Mn}$ atoms in the alloy. Differences in the degree of localization of the moments in CMS and CMA films are discussed with respect to calculations of the PDOS for these two systems. We show that the Co moment has more itinerant character in the CMA sample than in the CMS film, suggesting a smaller gap in the Co minority-spin band for CMA. These results are consistent with the measured weaker spin polarization in the CMA films compared to CMS. ${ }^{6}$ This demonstrates the predicted dominant role of the Co-Co interaction on the overall minority-spin gap and hence HMF.

\section{EXPERIMENT}

Epitaxial (001) orientated CMS and CMA layers were prepared on Cr-buffered $\mathrm{MgO}(001)$ substrates at ambient temperature using inductively coupled plasma (ICP) assisted magnetron sputtering. A composition-adjusted sputtering target $(43.7 \% \mathrm{Co}, 27.95 \% \mathrm{Mn}$, and $28.35 \% \mathrm{Si})$ was used to achieve stoichiometric CMS films, and a Co-Mn-Al (43.7\% $\mathrm{Co}, 27.95 \% \mathrm{Mn}$, and $28.35 \% \mathrm{Al}$ ) composition-adjusted target was used for the CMA films. The alloy layers were subsequently in situ annealed in order to provide optimum site ordering with either the $L 2_{1}$ structure (CMS films) or $B 2$ 
(a)

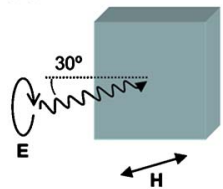

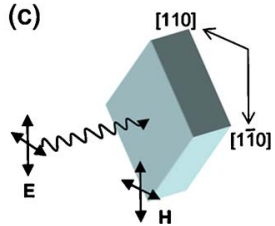

FIG. 1. (Color online) Schematics showing the various geometries used for the measurements (a) XMCD, [(b) and (c)] XMLD along the $\langle 100\rangle$ and $\langle 110\rangle$ crystal axes.

structure (CMA films). Following annealing, a $1.3 \mathrm{~nm} \mathrm{Al}$ capping layer was deposited to prevent oxidation. The crystal structure of the prepared Heusler alloy thin films was verified by $\mathrm{x}$-ray diffraction with in-plane and out-of-plane geometries. Full details of the sample characterization can be found elsewhere. ${ }^{6}$

X-ray absorption (XA) spectroscopy and XMCD measurements were performed at the Advanced Light Source on beamlines 6.3 .1 and 4.0.2 using circularly polarized soft $\mathrm{x}$-rays. The XA spectra were collected using the total electron yield (TEY) method of detection. This method is effective for obtaining interface-specific measurements for Heusler films which can be different to the bulk properties. ${ }^{13}$ The sample was inclined at an angle of $30^{\circ}$ to the $\mathrm{x}$-ray beam and the data collected at fixed photon helicity by reversing a magnetic field of 500 Oe along the x-ray beam, for each photon energy point in the scan [Fig. 1(a)].

The XMLD measurements were obtained on beamline 4.0.2 using an eight-pole electromagnet. ${ }^{14}$ The XA was measured in TEY from the (001) sample aligned with the $\mathrm{X}$-ray polarization vector along the in-plane [100] film axis as shown in Fig. 1(b). At each point in the scan the magnetic field was applied parallel and then perpendicular to the polarization vector, i.e., along [010]. In order to improve the electron yield signal, the magnetic field was canted slightly out of the sample surface. However, this does not affect the XMLD measurement as the surface normal represents a hard magnetic axis in these samples. The measurement was then repeated with the polarization vector rotated by $90^{\circ}$ to lie along the [010] sample axis. Since the Heusler films studied here have a cubic structure, the XMLD spectra obtained by rotation of either the $\mathrm{x}$-ray polarization vector, or the magnetization direction, should be the same. ${ }^{12}$ Therefore the final XMLD spectrum was obtained by averaging these two dichroism spectra in order to remove any nondichroic artifacts. The sample was then rotated by $45^{\circ}$ so that the polarization vector was parallel to the [110] or $[1 \overline{1} 0]$ axes, as illustrated in Fig. 1(c), and the XMLD obtained as above.

\section{RESULTS AND DISCUSSION}

The XMCD measured across the Co $L_{2,3}$ absorption edge for the CMS and CMA film is shown in Fig. 2(a), together with that obtained from a Co reference film capped with Al. The multiplet structure in the CMS film can be clearly seen and is similar to that observed previously for this material. ${ }^{15,16}$ The observation of this multiplet structure is indicative of a degree of localized character to the Co mo-
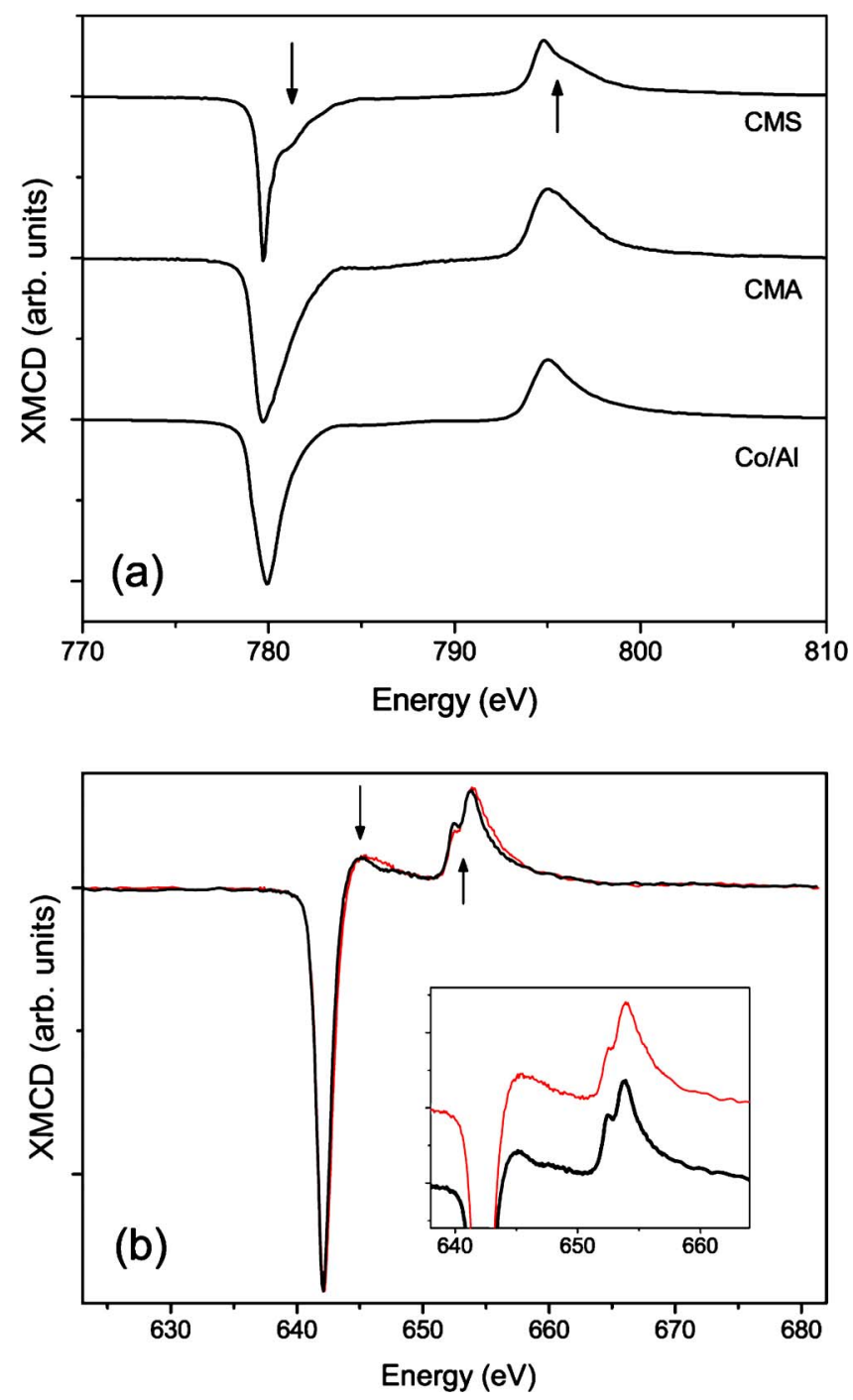

FIG. 2. (Color online) XMCD measured across (a) Co $L_{2,3}$ and (b) $\mathrm{Mn} L_{2,3}$ absorption edges. In (a) the spectra from the $\mathrm{Co}_{2} \mathrm{MnSi}$ and $\mathrm{Co}_{2} \mathrm{MnAl}$ films are compared to a $\mathrm{Co} / \mathrm{Al}$ reference sample. In (b) the Mn spectra obtained for CMS (solid black line) and CMA (thin red line) are overlaid. The arrows indicate positions of specific multiplet structure. The inset shows an enlargement with the CMS and CMA spectra offset vertically for clarity.

ment and originates from $2 p-3 d$ and $3 d-3 d$ Coulomb and electrostatic interactions. ${ }^{17}$ In contrast, the spectrum obtained from the CMA film more closely resembles the $\mathrm{Co} / \mathrm{Al}$ reference film. The corresponding XMCD spectra measured around the $\mathrm{Mn} L_{2,3}$ are shown in Fig. 2(b). Again a clear multiplet structure can be seen in the XMCD from the CMS film and is similar to spectra obtained from a range of Heusler alloys containing $\mathrm{Mn} .^{15,16,18-21}$ However, unlike the Co $\mathrm{XMCD}$ there is evidence of multiplet structure in the $\mathrm{Mn}$ XMCD from the CMA film (note the feature at the $L_{2}$ absorption edge), although of weaker intensity than for the CMS film.

These results suggest that while the Co moment has more itinerant character in the CMA film, the Mn moment has a degree of localization for both materials. The reason for this difference can be understood by considering the partial den- 


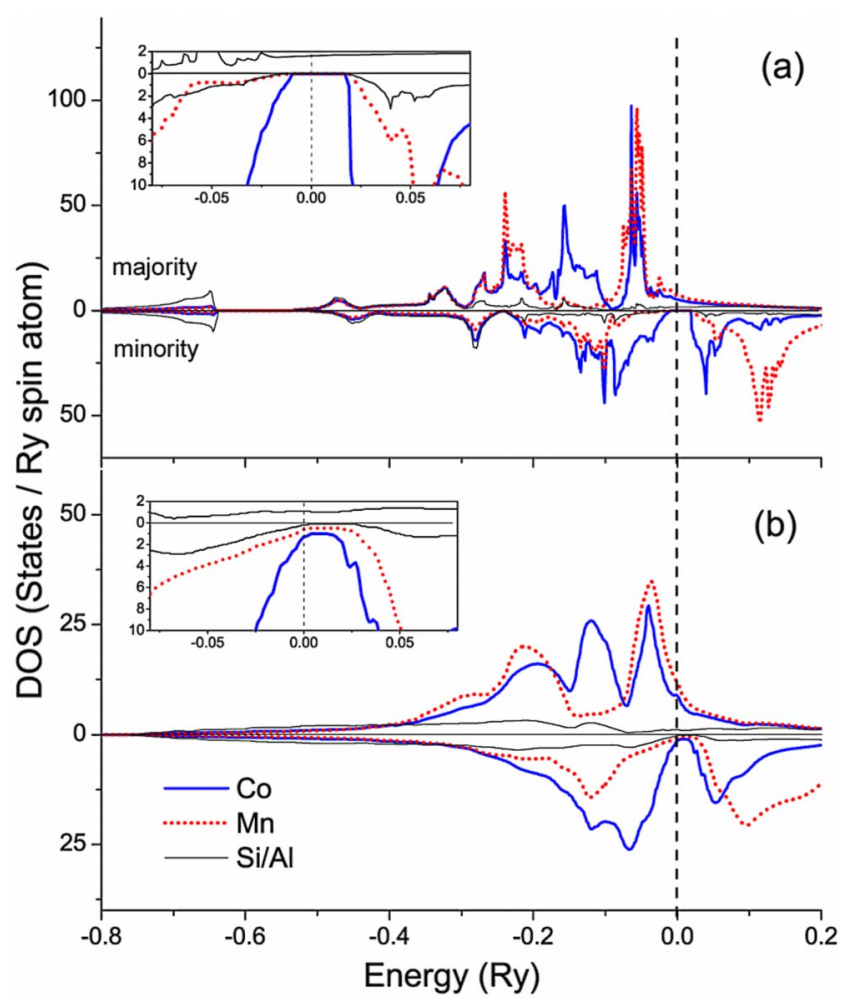

FIG. 3. (Color online) The calculated partial density of states for $\mathrm{Co}, \mathrm{Mn}$, and $\mathrm{Al} / \mathrm{Si}$ in the (a) $\mathrm{Co}_{2} \mathrm{MnSi} L 2_{1}$ structure and the (b) $\mathrm{Co}_{2} \mathrm{MnAl} B 2$ structure. The insets show an enlargement of the PDOS around the Fermi level for each structure.

sity of states. For CMS films, the Fermi level lies in the middle of the overall minority-spin gap such that there is an absence of minority-spin DOS for both Co and Mn atoms. This can be seen in the calculated PDOS for the CMS film in the $L 2_{1}$ structure, as shown in Fig. 3(a). ${ }^{6}$ Hence the exclusion of minority spin should occur around both Co and Mn atoms leading to local moment formation on both sites, in agreement with the XMCD from this film. The calculated PDOS for the CMA film in the $B 2$ structure is shown in Fig. 3(b). ${ }^{6}$ In this case the Fermi level is positioned close to the edge of the overall gap. Since the Co band gap is narrower than the Mn gap, this leads to a finite minority-spin Co DOS at the Fermi level, as seen in the inset to Fig. 3(b). The Mn minority-spin DOS is closer to zero at $E_{F}$ resulting in $\mathrm{Mn}$ moments with more local character. However even for the Mn sites, the minority-spin gap in CMA is less well defined than in CMS. This would explain the weaker multiplet features in the Mn XMCD from the CMA sample, owing to a reduced localization of the Mn moment. It should also be noted that although CMA in the $B 2$ structure is discussed here, calculations of the PDOS for $L 2_{1}$ ordered CMA yield a similar weak minority-spin gap,${ }^{6}$ in contrast to the results calculated for CMS films.

Previous authors have suggested that the fine structure in the Co and Mn XMCD is related to the detailed shape of the unoccupied states in the PDOS. ${ }^{21}$ From these arguments the more metallic appearing Co XMCD seen here for the CMA film could be explained in terms of the broader DOS obtained in the $B 2$ structure as opposed to the $L 2_{1}$ structure of

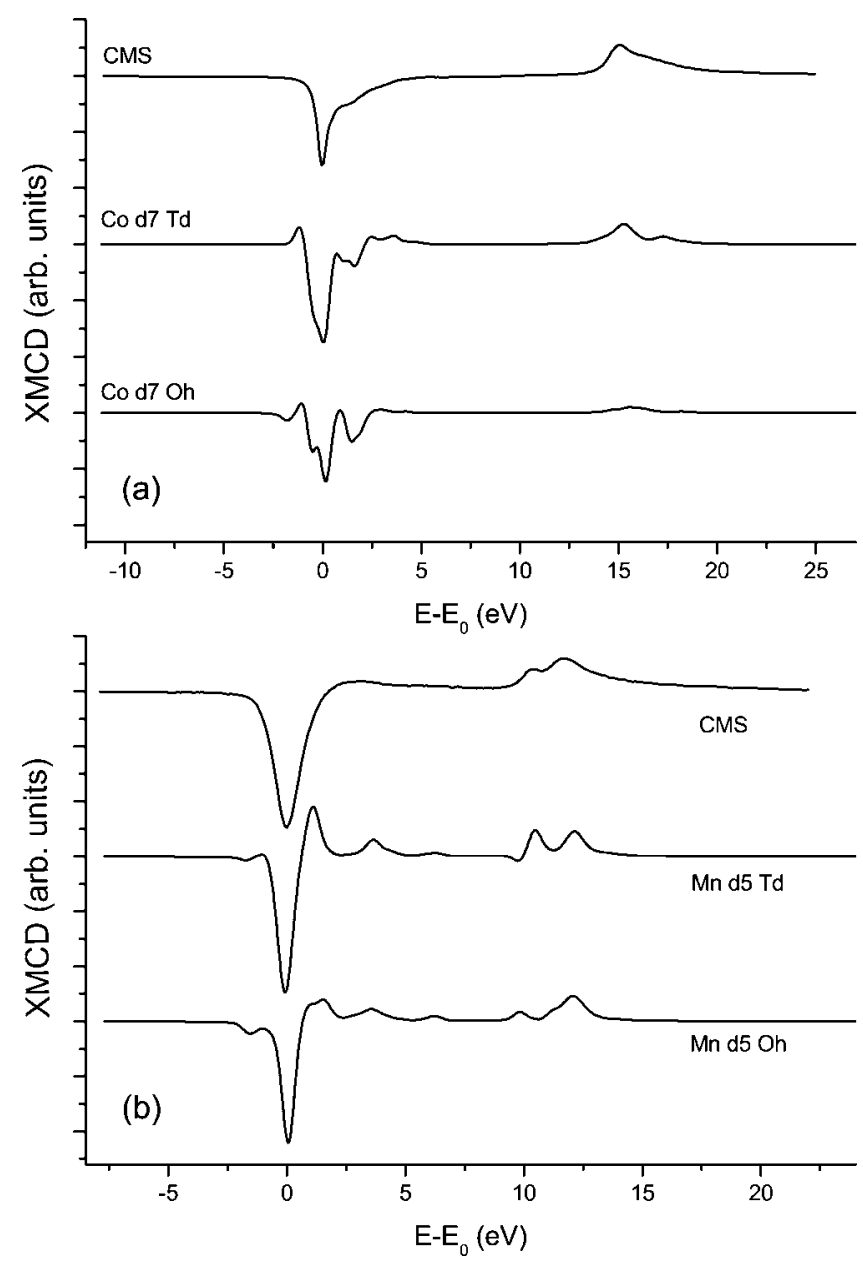

FIG. 4. (a) XMCD recorded across the Co $L_{2,3}$ absorption edges for $\mathrm{Co}_{2} \mathrm{MnSi}$ (top curve) and multiplet calculations for atomic $\operatorname{Co} d^{7}$ in tetrahedral $\left(T_{d}\right)$ and octahedral $\left(O_{h}\right)$ crystal field. (b) XMCD recorded across the $\mathrm{Mn} L_{2,3}$ absorption edges (top curve) and multiplet calculation for atomic $\mathrm{Mn} d^{5}$ in tetrahedral $\left(T_{d}\right)$ and octahedral $\left(O_{h}\right)$ crystal fields.

CMS films (Fig. 3). ${ }^{15,21}$ However there are two principal objections to these arguments: (i) calculations of the XMCD spectra based purely on band-structure considerations do not accurately reproduce the fine structure in the Co XMCD (Ref. 21); and (ii) the PDOS calculated for both Co and Mn unoccupied states are broader for the $B 2$ ordered CMA film than for the $L 2_{1}$ CMS film, and yet the width of the main $\mathrm{XMCD}$ peaks and fine-structure features are comparable for the two Mn XMCD spectra shown in Fig. 2(b).

The XMCD spectra obtained across the Co and $\mathrm{Mn} L_{2,3}$ absorption edges are compared to atomic multiplet calculations in Fig. 4. The theoretical spectra were obtained using atomic multiplet calculations following the method described in Ref. 22. The $L_{2,3}$ XA spectra were obtained from the electric-dipole allowed transitions between the ground state $3 d^{n}$ and the final-state $2 p^{5} 3 d^{n+1}$ configurations. The octahedral crystal field was $10 D q=1.0 \mathrm{eV}$ and the tetrahedral crystal field was -0.5 and $-0.6 \mathrm{eV}$, for $\mathrm{Mn}$ and $\mathrm{Co}$, respectively. The calculated results were broadened by a Lorentzian of $\Gamma=0.1(0.3) \mathrm{eV}$ for the $L_{3}\left(L_{2}\right)$ edge to account for intrinsic 
linewidth broadening and a Gaussian of $\sigma=0.2 \mathrm{eV}$ for the instrumental broadening.

It can be seen from Fig. 4(a) that the Co XMCD spectrum bears some similarity to the calculated spectra for localized divalent Co atoms. The strong Co-Co and Co-Mn hybridization leads to a smearing of the multiplet features in the experimental spectra compared to the calculations. This makes direct comparisons difficult, although the calculation using a tetrahedral crystal field appears to give a better match with the data. This is consistent with the expected crystal symmetry in the full Heusler alloy structure where the Co atoms are in tetrahedral sites with respect to the first neighbor Mn atoms. However the second-neighbor Co-Co interactions are also important in the full Heusler alloys, and this leads to octahedral crystal-field splitting. ${ }^{2,3}$ The Mn XMCD spectrum [Fig. 4(b)] shows some of the fine structure produced in the multiplet calculation for $\mathrm{Mn} d^{5}$, most notably around the $L_{2}$ absorption edge. In this case the octahedral crystal-field calculation seems closer to the experimental curve. However the features around the $L_{3}$ absorption edge are not well reproduced in the calculation for either crystal-field symmetry, suggesting an alternative or possibly mixed-valence state could occur for Mn in the CMS film.

The XA and XMLD spectra obtained from the CMS film around the Mn $L_{2,3}$ absorption edges are shown in Fig. 5 for measurements obtained along the $\langle 100\rangle$ and $\langle 110\rangle$ directions. It can be seen that a reasonably substantial linear dichroism effect is obtained in this sample $(\sim 4.5 \%$, taken as the ratio of the maximum XMLD to the $L_{3}$ absorption peak height). Although the size of the effect is not as large as that observed in localized charge systems, such as diluted $\mathrm{Mn}^{2+}$ ions in GaAs, ${ }^{11}$ it is still several times larger than would be anticipated for a metallic system. Further, the anisotropy in the XMLD that was found in Ref. 11 is also clearly evident here as can be seen by the changing spectral shape for measurements along the two sample axes. We also note that despite differences in the individual XMLD scans obtained here and their counterparts in the previous paper ${ }^{11}$ the mean spectra are very similar.

The much larger XMLD feature at the $L_{2}$ absorption edge seen in Fig. 5 compared to the previous work ${ }^{11}$ is due to the greater intensity of the $L_{2}$ XAS peak in the CMS film compared to a localized charge system (e.g., a metallic oxide). In fact the relative intensities of the XMLD at the $L_{3}$ and $L_{2}$ absorption edges are similar to those seen in metallic systems. ${ }^{23,24}$ The corresponding XA and XMLD spectra obtained around the Co $L_{2,3}$ absorption edges of the CMS film are shown in Fig. 6. A strong dichroism effect is also seen at these absorption edges $(\sim 6 \%)$. This effect is approximately three times larger than in pure metallic Co films. ${ }^{23,24}$ To our knowledge these are the first observations of an enhanced XMLD in a half-metallic alloy, and indicate a degree of localization of the Co and $\mathrm{Mn}$ moments. Unlike the Mn absorption edges at the Co edges there is little apparent anisotropy in the linear dichroism, resulting in a nearly vanishing mean XMLD curve.

The XA and XMLD spectra measured at the Mn and Co absorption edges for the CMA sample are shown in Figs. 7 and 8, respectively. Again a strong anisotropy is seen in the Mn XMLD (Fig. 7) although the magnitude of the dichroism

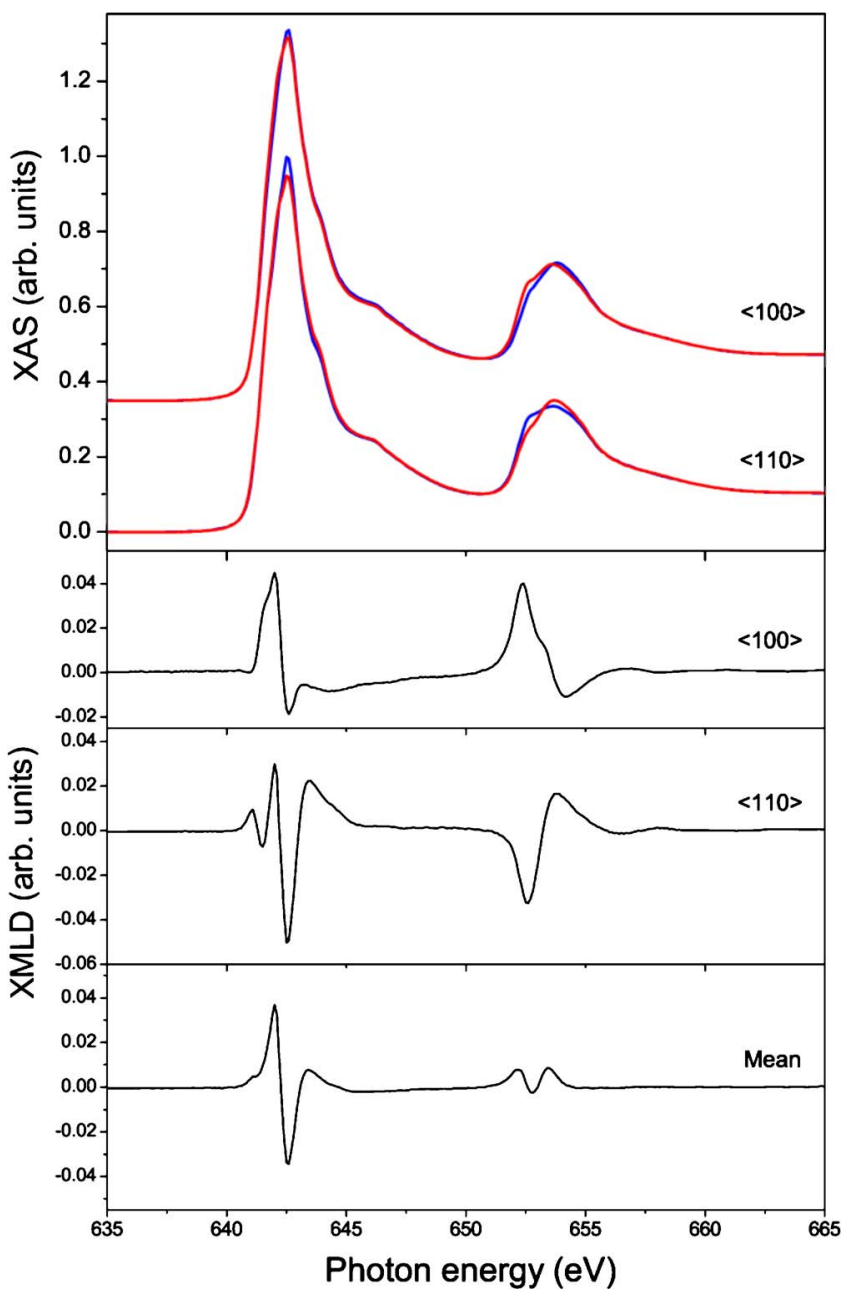

FIG. 5. (Color online) $\mathrm{Mn} L_{2,3}$ spectra for $\mathrm{Co}_{2} \mathrm{MnSi}$ film. The $\mathrm{x}$-ray absorption (XA) spectra (top) measured along the $\langle 100\rangle$ and $\langle 110\rangle$ crystal axes. The red and blue curves correspond to measurements made with the magnetic field applied parallel and perpendicular to the linear polarization vector. The XA spectra are normalized to 1 at the $L_{3}$ maximum and are displaced vertically for clarity. The corresponding XMLD spectra obtained by subtraction of the two XA curves for each crystal direction together with the mean of these spectra, are shown underneath.

is reduced by a factor of $\sim 1.8$. For the Co XMLD spectra (Fig. 8), the reduction factor is $\sim 3.6$ and a slight difference can be seen between the $\langle 100\rangle$ and $\langle 110\rangle$ directions. By applying a standard sum-rule analysis ${ }^{25}$ to the measured XMCD spectra, the ratios of the total $\mathrm{Co}$ and $\mathrm{Mn}$ atomic moments between the CMS and CMA samples, i.e., the values of $\mu_{\mathrm{Co}}^{\mathrm{CMS}} / \mu_{\mathrm{Co}}^{\mathrm{CMA}}$ and $\mu_{\mathrm{Mn}}^{\mathrm{CMS}} / \mu_{\mathrm{Mn}}^{\mathrm{CMA}}$ were determined to be $\sim 1.52$ and $\sim 1.25$, respectively. This calculation assumes that the number of $3 d$ holes and the degree of $\mathrm{Mn} 2 p j$ $=3 / 2$ and $j=1 / 2$ mixing ${ }^{10}$ are the same for both samples. Previous theoretical calculations of these moments ${ }^{26}$ give values for these ratios of $\sim 1.34$ and $\sim 1.16$, which are smaller but in consistent proportions to the values determined here. As the XMLD intensity is proportional to the expectation value of the atomic moment, ${ }^{11,12,23,24}$ the scaling factor of the XMLD between the CMS and CMA samples due purely to the measured atomic moment ratios (above) 


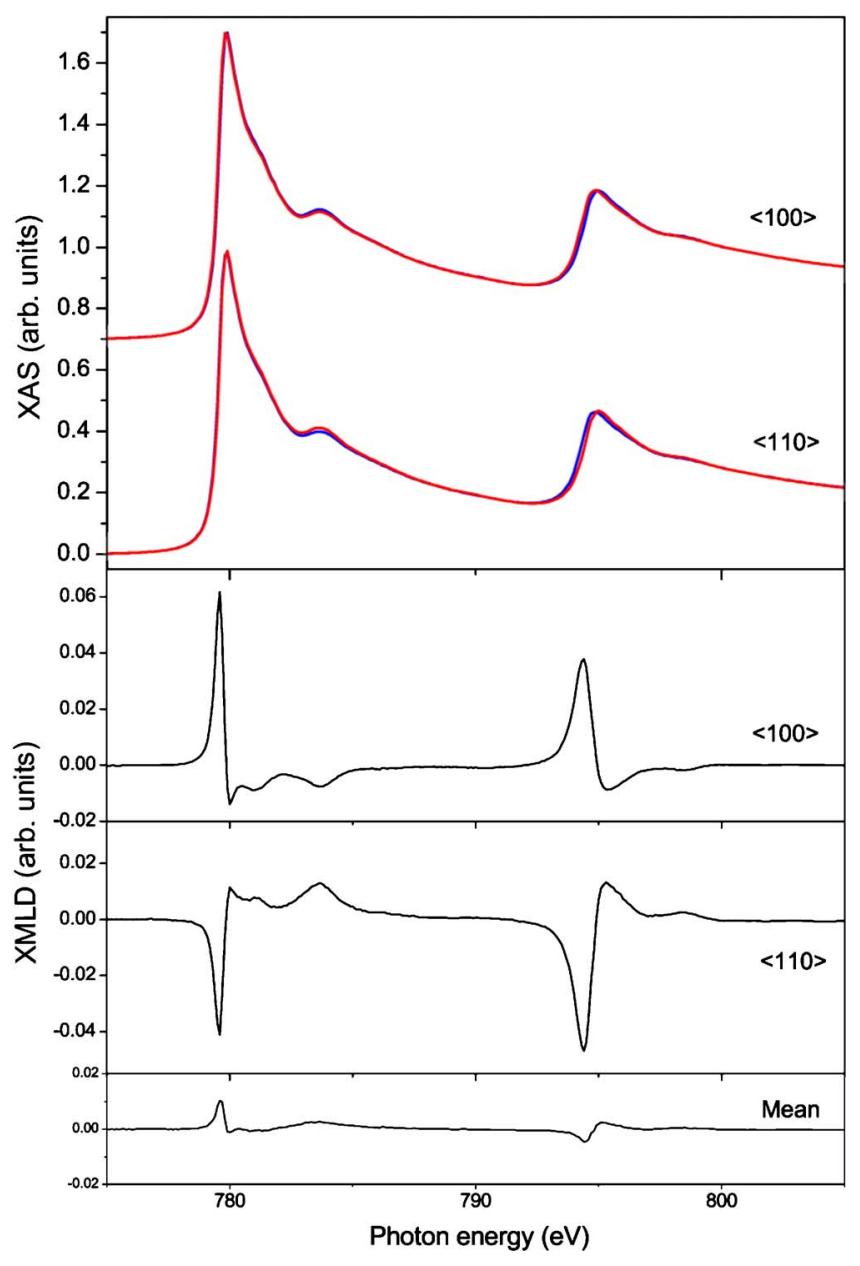

FIG. 6. (Color online) Co $L_{2,3}$ spectra for $\mathrm{Co}_{2} \mathrm{MnSi}$ film. Details as for Fig. 5 .

would be $\sim 2$ for Co and $\sim 1.5$ for Mn. While this accounts for most of the change in the Mn XMLD, it is only about half of the change seen in the Co XMLD. The origin of the remaining reduction in the Co XMLD must therefore be the change in the degree of localization of the Co moment, as suggested from the XMCD measurements.

Further evidence to support this conclusion can be found by comparing the lineshape of the XMLD (measured along the $\langle 100\rangle$ direction) from the two Heusler samples and a $\mathrm{FeCo} / \mathrm{GaAs}$ thin-film standard sample, ${ }^{27}$ as shown in Fig. 9. It can be seen that the spectrum from the CMA sample closely resembles that of the metallic FeCo film, whereas the CMS spectrum comprises sharper features. Thus it appears that the Co moments in the CMA film are more metallic in nature than those of the CMS sample. However, as can be seen by comparing Fig. 8 and Fig. 6, the anisotropy in the Co XMLD is greater in the CMA film than the CMS sample. This could be related to the different crystal structures of the Heusler alloys, $B 2$ for CMA and $L 2_{1}$ for CMS, resulting in different crystal-field symmetries. Further to the measurements presented here, it was found that the anisotropy in the Co XMLD was even greater in a CMA sample measured in the as-prepared (and thus least ordered) state. These results will be presented elsewhere. The survival of the crystal-field splitting of the empty $d$ states depends on the Co-Co and

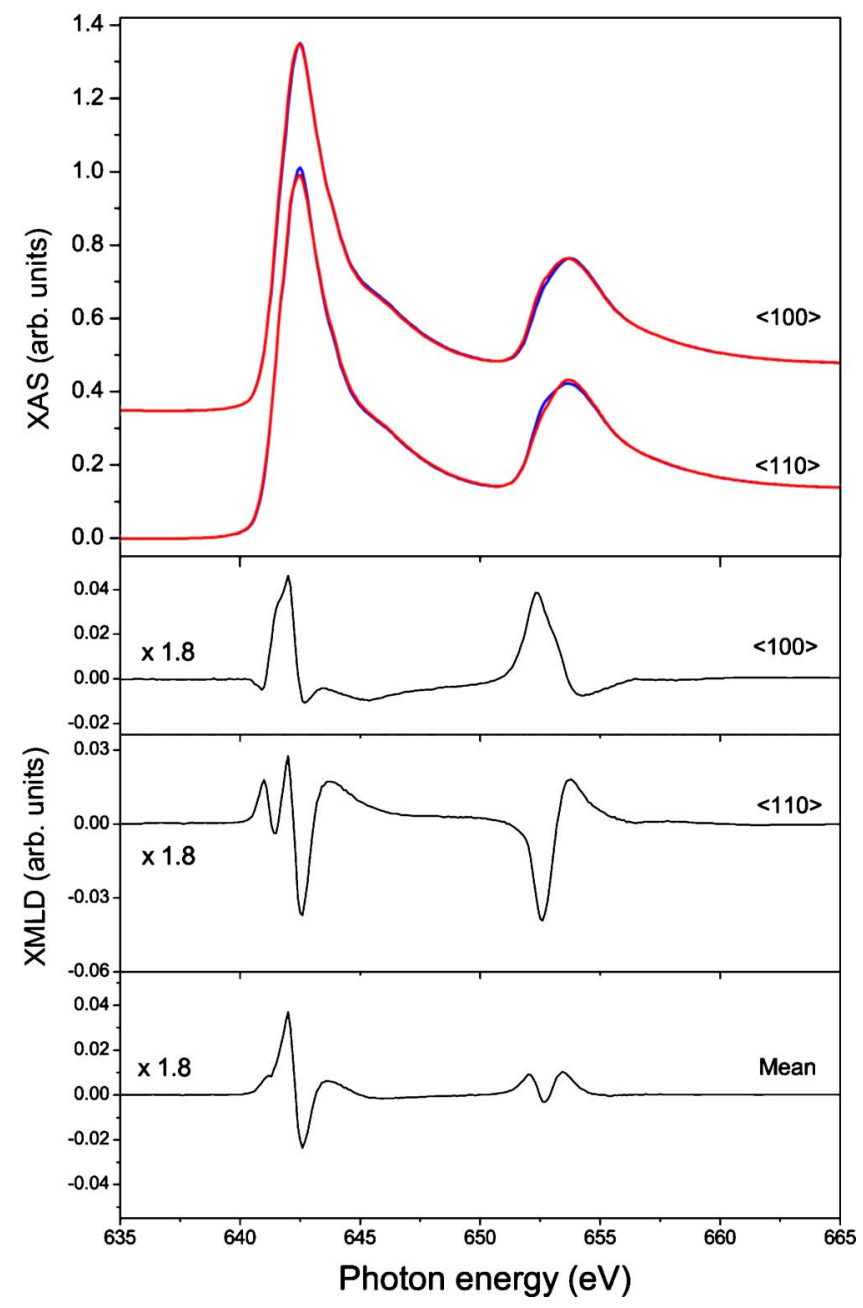

FIG. 7. (Color online) $\mathrm{Mn} L_{2,3}$ spectra for $\mathrm{Co}_{2} \mathrm{MnAl}$ thin film. Details as for Fig. 5.

Co-Mn hybridization which lies at the heart of the formation of the minority-spin gap in the full Heusler alloys. XMLD may therefore offer a unique method for probing subtle changes in the interatomic hybridization in Heusler alloys via changes in the crystal-field symmetry.

\section{CONCLUSION}

In summary, we have utilized x-ray magnetic circular and linear dichroism measurements across the $L_{2,3}$ absorption edges to probe the localized character of the $\mathrm{Co}$ and $\mathrm{Mn}$ interface moments in half-metallic Heusler films. A multiplet fine structure was found in the XMCD curves, and an enhanced X-ray magnetic linear dichroism was observed in the $\mathrm{Co}_{2} \mathrm{MnSi}$ sample for both $\mathrm{Co}$ and $\mathrm{Mn}$ absorption edges. While these two characteristics were largely retained at the $\mathrm{Mn}$ absorption edges in the $\mathrm{Co}_{2} \mathrm{MnAl}$ sample, they were both reduced at the $\mathrm{Co}$ absorption edges. These measurements suggest that although the Mn moments in both Heusler alloys show some localized character, the Co moments are less well localized in CMA. For the latter case the calculated PDOS yields a larger effective minority-spin gap for Mn than for Co states. Thus these results indicate an important connec- 


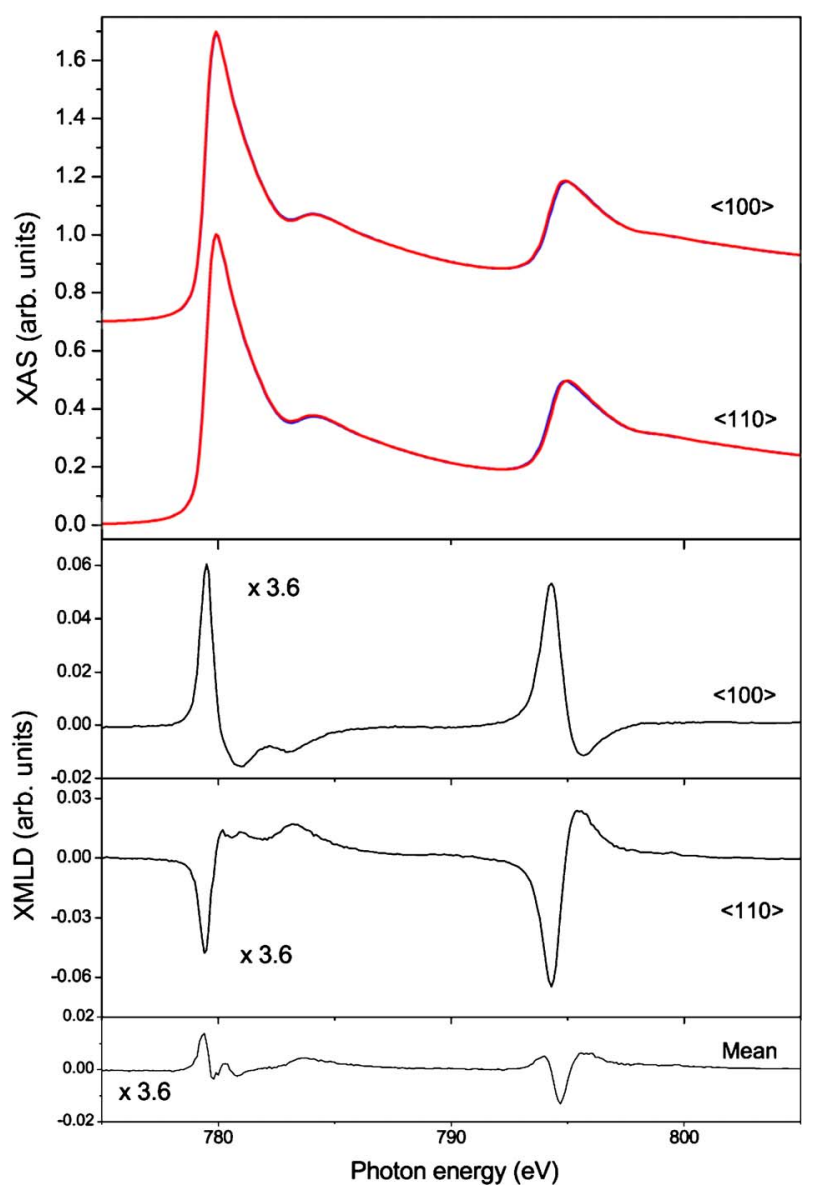

FIG. 8. (Color online) Co $L_{2,3}$ spectra for $\mathrm{Co}_{2} \mathrm{MnAl}$ thin film. Details as for Fig. 5.

tion between the minority-spin gap and the degree of localization of the Co and Mn moments. As the overall band gap is determined by the Co PDOS, the observation of localized Co moments by the X-ray methods used here should coincide

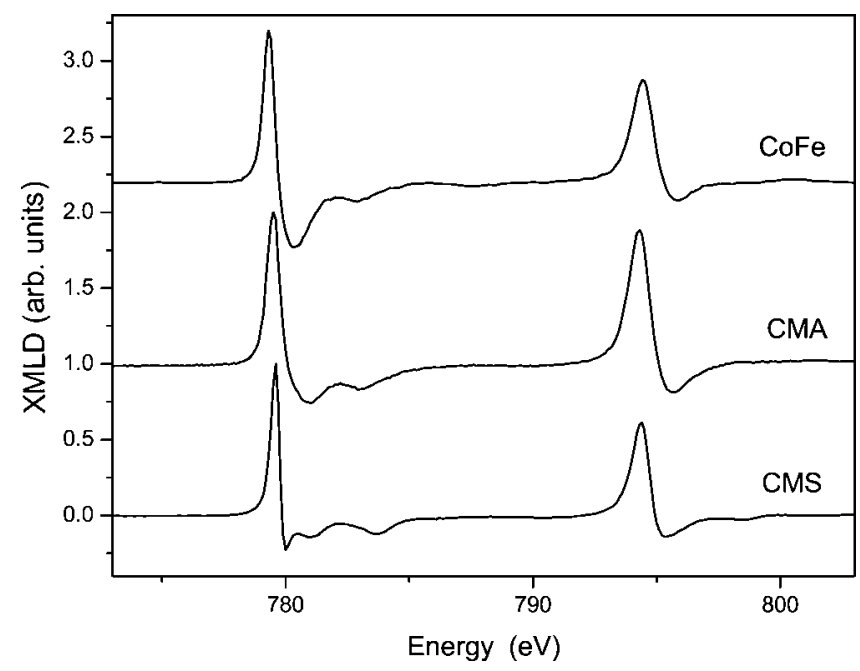

FIG. 9. XMLD at the Co $L_{2,3}$ absorption edges measured along the $\langle 100\rangle$ crystal axes for $\mathrm{Co}_{2} \mathrm{MnSi}$ and $\mathrm{Co}_{2} \mathrm{MnAl}$ compared to a reference sample of $\mathrm{FeCo} / \mathrm{GaAs}$. The XMLD is normalized to 1 at the $L_{3}$ maximum. The curves are displaced vertically for clarity.

with the onset of half-metallic behavior. In fact, magnetotransport measurements on these samples yielded a significantly larger spin-polarization for the CMS film ${ }^{28}$ compared to the CMA sample, in agreement with the results presented here.

\section{ACKNOWLEDGMENTS}

The Advanced Light Source is supported by the Director, Office of Science, Office of Basic Energy Sciences, of the U.S. Department of Energy under Contract No. DE-AC0205CH11231. A part of this work was supported by the Grant Program of the New Energy and Industrial Development Organization (NEDO) and by a Research Foundation for Young Scientists from the Japan Society for the Promotion of Science (JSPS).
${ }^{1}$ R. A. de Groot, F. M. Mueller, P. G. van Engen, and K. H. J. Buschow, Phys. Rev. Lett. 50, 2024 (1983).

${ }^{2}$ I. Galanakis, P. H. Dederichs, and N. Papanikolaou, Phys. Rev. B 66, 174429 (2002).

${ }^{3}$ I. Galanakis, Ph. Mavropoulos, and P. H. Dederichs, J. Phys. D 39, 765 (2006).

${ }^{4}$ H. C. Kandpal, G. H. Fecher, and C. Felser, J. Phys. D 40, 1507 (2007).

${ }^{5}$ Y. Sakuraba, J. Nakata, M. Oogane, H. Kubota, Y. Ando, A. Sakuma, and T. Miyazaki, Jpn. J. Appl. Phys., Part 2 44, L1100 (2005).

${ }^{6}$ M. Oogane, Y. Sakuraba, J. Nakata, H. Kubota, Y. Ando, A. Sakuma, and T. Miyazaki, J. Phys. D 39, 834 (2006).

${ }^{7}$ Y. Sakuraba, M. Hattori, M. Oogane, Y. Ando, H. Kato, A. Sakuma, H. Kubota, and T. Miyazaki, Appl. Phys. Lett. 88, 192508 (2006).

${ }^{8}$ J. Kübler, A. R. Williams, and C. B. Sommers, Phys. Rev. B 28,
1745 (1983).

${ }^{9}$ P. Gambardella, S. S. Dhesi, S. Gardonio, C. Grazioli, P. Ohresser, and C. Carbone, Phys. Rev. Lett. 88, 047202 (2002).

${ }^{10}$ K. W. Edmonds, N. R. S. Farley, T. K. Johal, G. van der Laan, R. P. Campion, B. L. Gallagher, and C. T. Foxon, Phys. Rev. B 71, 064418 (2005)

${ }^{11}$ A. A. Freeman, K. W. Edmonds, G. van der Laan, N. R. S. Farley, T. K. Johal, E. Arenholz, R. P. Campion, C. T. Foxon, and B. L. Gallagher, Phys. Rev. B 73, 233303 (2006).

${ }^{12}$ G. van der Laan, E. Arenholz, R. V. Chopdekar, and Y. Suzuki, Phys. Rev. B 77, 064407 (2008); E. Arenholz, G. van der Laan, R. V. Chopdekar, and Y. Suzuki, ibid. 74, 094407 (2006); Phys. Rev. Lett. 98, 197201 (2007).

${ }^{13}$ M. Kallmayer, H. Schneider, G. Jakob, H. J. Elmers, K. Kroth, H. C. Kandpal, U. Stumm, and S. Cramm, Appl. Phys. Lett. 88, 072506 (2006).

${ }^{14}$ E. Arenholz and S. O. Prestemon, Rev. Sci. Instrum. 76, 083908 
(2005).

${ }^{15}$ J. Schmalhorst, A. Thomas, S. Kämmerer, O. Schebaum, D. Ebke, M. D. Sacher, G. Reiss, A. Hütten, A. Turchanin, A. Gölzhäuser, and E. Arenholz, Phys. Rev. B 75, 014403 (2007).

${ }^{16}$ N. D. Telling, P. S. Keatley, G. van der Laan, R. J. Hicken, E. Arenholz, Y. Sakuraba, M. Oogane, Y. Ando, and T. Miyazaki, Phys. Rev. B 74, 224439 (2006).

${ }^{17}$ B. T. Thole and G. van der Laan, Phys. Rev. B 38, 3158 (1988).

${ }^{18}$ T. Saito, T. Katayama, T. Ishikawa, M. Yamamoto, D. Asakura, and T. Koide, Appl. Phys. Lett. 91, 262502 (2007).

${ }^{19}$ A. Kimura, S. Suga, T. Shishidou, S. Imada, T. Muro, S. Y. Park, T. Miyahara, T. Kaneko, and T. Kanomata, Phys. Rev. B 56, 6021 (1997).

${ }^{20}$ K. Miyamoto, K. Ioria, A. Kimuraa, T. Xiea, M. Taniguchia, B. S. Qiaob, and K. Tsuchiyac, Solid State Commun. 128, 163 (2003).
${ }^{21}$ V. N. Antonov, O. Jepsenb, A. N. Yaresko, and A. P. Shpak, J. Appl. Phys. 100, 043711 (2006).

${ }^{22}$ G. van der Laan and B. T. Thole, Phys. Rev. B 43, 13401 (1991).

${ }^{23}$ M. M. Schwickert, G. Y. Guo, M. A. Tomaz, W. L. O'Brien, and G. R. Harp, Phys. Rev. B 58, R4289 (1998).

${ }^{24}$ S. S. Dhesi, G. van der Laan, E. Dudzik, and A. B. Shick, Phys. Rev. Lett. 87, 067201 (2001).

${ }^{25}$ C. T. Chen, Y. U. Idzerda, H.-J. Lin, N. V. Smith, G. Meigs, E. Chaban, G. H. Ho, E. Pellegrin, and F. Sette, Phys. Rev. Lett. 75, 152 (1995); B. T. Thole, P. Carra, F. Sette, and G. van der Laan, ibid. 68, 1943 (1992).

${ }^{26}$ I. Galanakis, Phys. Rev. B 71, 012413 (2005).

${ }^{27}$ E. Arenholz, Y. U. Idzerda, and G. van der Laan (unpublished). ${ }^{28}$ Y. Sakuraba, M. Hattori, M. Oogane, Y. Ando, H. Kubota, and T. Miyazaki (unpublished). 